\title{
UN ANÁLISIS COMPARATIVO DEL USO DE DIAGRAMAS EN DOS PRÁCTICAS MATEMÁTICAS DE LA ANTIGÜEDAD
}

\author{
MAnuel J. GARCíA-PÉREZ \\ Universidad de Sevilla \\ Departamento de Lógica y Filosofía de la Ciencia \\ manueljgarciaperez@gmail.com \\ Tamires Dal Magro \\ Universidade Estadual de Campinas (UNICAMP) \\ Instituto de Filosofia e Ciências Humanas \\ tamiresdma@gmail.com
}

RESUMEN: En nuestro artículo, analizaremos las diferencias y similitudes que podemos encontrar en el uso de diagramas entre dos prácticas matemáticas de la antigüedad. Mostraremos que no existe un solo uso posible de éstos, sino que tienen que considerarse como herramientas de razonamiento creadas y manipuladas de acuerdo con los propósitos de cada tradición. Argumentaremos que las diferencias en los procedimientos y organizaciones del conocimiento que ambas tradiciones presentan no implican ningún tipo de valoración acerca de su mayor o menor "matematicidad", ya que ambas tradiciones llegaron a resultados matemáticos suficientemente generales y precisos, hecho que es característico de investigaciones matemáticas a un nivel teórico.

PALABRAS CLAVE: análisis contextual, historia de la geometría, razonamiento diagramático, teorema de Pitágoras, procedimiento gou gu

SUMMARY: In our article, we will analyze the differences and similarities that we can find in the use of diagrams between two mathematical practices of antiquity. We will show that there is no single possible use of these, but that they have to be considered as reasoning tools created and manipulated according to the purposes of each tradition. We will argue that the differences in the procedures and organizations of knowledge that both traditions present do not imply any kind of assessment of their greater or lesser "mathematicity", since both traditions reached sufficiently general and precise mathematical results, a fact that is characteristic of mathematical research at a theoretical level.

KEY WORDS: contextual analysis, history of geometry; diagrammatic reasoning, Pythagorean Theorem, gou gu procedure

\section{Introducción}

En este artículo se analizará comparativamente cómo dos prácticas matemáticas de la antigüedad hicieron uso de los diagramas en relación con la búsqueda y establecimiento de resultados matemáticos. 
Para ello, centraremos nuestra atención tanto en los Elementos de Euclides como en el Zhou bi (Zhou bi suanjing) y los Nueve capítulos de los procedimientos matemáticos (Jiuzhang suanshu). ${ }^{1}$

Con esto no queremos decir que estemos representando o analizando toda la matemática griega o china de la antigüedad; por ejemplo, existen diferencias entre la práctica matemática presentada en los Elementos de la que presenta Autolico en Sobre las esferas en movimiento, o Arquímedes en Sobre las espirales (Heath 1921; Netz 1999). Nuestro propósito es menos general, se centra en comparar cómo en las obras que mencionamos al inicio de este trabajo se hizo uso de los diagramas para demostrar el "Teorema de Pitágoras", el cual es conocido en las matemáticas chinas como "Procedimiento gou gu".

Una de las razones que hace a este caso de estudio interesante se relaciona con las afirmaciones que usualmente historiadores de las matemáticas, o matemáticos, han hecho acerca de la poca importancia de las matemáticas "orientales" frente a las occidentales. ${ }^{2}$ A veces, se afirma que estas matemáticas suelen centrar su atención en la búsqueda de soluciones a problemas meramente prácticos, ${ }^{3}$ sin alcanzar, por lo tanto, el rigor deductivo o naturaleza puramente matemática que puede encontrarse en los trabajos de la antigua Grecia. Un caso llamativo que sirve para ilustrar esta situación es el del matemático Hardy, quien llegó a afirmar que "los griegos fueron los primeros matemáticos que aún son 'reales' para nosotros hoy día. Las matemáticas orientales pueden ser una curiosidad interesante, pero las matemáticas griegas son lo real" (Hardy 1940/1992, p. 12).

${ }^{1}$ Todas las citas en nuestro trabajo de los Elementos hacen referencia a la traducción inglesa de Heath (1956) y a la traducción en castellano de Puertas Castaños (2000). En cuanto al Zhou bi, usamos la traducción al inglés de Cullen (1996), y respecto a los Nueve capítulos de los procedimientos matemáticos — de aquí en adelante, Nueve capítulos - la traducción al francés de Chemla y Guo (2004).

${ }^{2}$ Para una presentación crítica general de este tipo de afirmaciones, véase Joseph 2011. Para una presentación específica de cómo se han comparado estas dos "tradiciones" matemáticas en cierto periodo histórico, véase Charette (2012). Dentro de las matemáticas "orientales" se ha tendido a incluir los desarrollos matemáticos que surgieron en lugares tan distantes y periodos históricos diferentes como las matemáticas babilónicas, egipcias, indias o chinas.

${ }^{3}$ Por ejemplo, si miramos algunos capítulos de los Nueve capítulos, podemos ver que el primero se titula "Medición de terrenos", el segundo "Soja y arroz", etc. Esto puede dar la impresión (errónea) de que éste era un tipo de desarrollo matemático de corte práctico. Chemla (2009) analiza esta situación en relación con la antigua civilización China y la supuesta concepción práctica de sus desarrollos matemáticos.

${ }^{4}$ Todas las citas, a menos que se indique lo contrario, son traducciones nuestras de su idioma original. 
Con afirmaciones de este tipo se asume que cualquier tipo de matemáticas alejadas del ideal griego se consideran de menor importancia histórica o meras curiosidades. De esta manera, la diversa y rica historia de las diferentes elaboraciones matemáticas se reduce a simplificaciones excesivas, en las que se busca una reconstrucción racional de la génesis y evolución del pensamiento matemático que no corresponde con su desarrollo "real"; esto es, histórico, culturalmente influenciado, contextual, etcétera.

Sin embargo, en la interpretación de Hardy podemos ver que, aunque las matemáticas orientales le resultan menos importantes, asume alguna característica distintiva en ellas que lo obligan a considerarlas también como matemáticas. Esto nos conduce a pensar que existe alguna característica común entre las diversas prácticas matemáticas, por distintos que sean sus desarrollos, métodos y herramientas de razonamiento, que nos permite caracterizarlas como genuinamente matemáticas.

En relación con este punto, nuestra propuesta es considerar la distinción que De Regt y Dieks (2005, pp. 138-139) presentan entre los niveles macro, meso y micro en el análisis de las prácticas científicas. Creemos que esta consideración teórica puede aplicarse de igual manera al análisis de las prácticas matemáticas, y permitiría dar una caracterización general de lo que éstas son sin que se ignore su variación histórico-cultural.

En el nivel macro necesitamos una caracterización del conocimiento matemático que sea lo suficientemente general como para poder reconocer los desarrollos y las prácticas actuales, así como los que emergieron en la antigüedad, pertenecientes a un mismo corpus de conocimiento. Dar una definición precisa de lo que son las matemáticas en este nivel es una tarea que excede los límites de este trabajo. Autores como Ferreirós (2016, pp. 28-34; pp. 40 y ss.) han intentado describirlas como un cuerpo de conocimiento que surge por expansiones sucesivas del estudio -mediante el uso de símbolosde números y/o formas; por otro lado, Joseph (2011) afirma que las matemáticas son "cualquier actividad que surja de, o genere directamente, conceptos relacionados con números o configuraciones espaciales, junto a alguna forma de [razonamiento] lógico" (p. 30). En nuestro trabajo comparativo será suficiente considerar, tal y como Ferreirós (2016, pp. 112-152) propone, que la transición de las técnicas prácticas en geometría - prácticas de medición o construcción de artefactos para la producción de dibujos - al desarrollo de un cuerpo teórico de conocimiento está vinculada principalmente con 
la emergencia de ciertos objetivos y valores en la propia práctica. La dirección hacia un cuerpo de conocimiento teórico en geometría y matemáticas, en general, está relacionada con la búsqueda de la exactitud y la generalización de los resultados.

El nivel meso tiene que ver con cómo las diferentes comunidades de matemáticos hacen matemáticas. En este nivel, cada comunidad puede desarrollar prácticas cualitativamente distintas que darían lugar a diversas organizaciones del conocimiento, guiadas por diferentes metas o valores - para las cuales podrán usar distintas herramientas de razonamiento, como fórmulas o diagramas-, y asumir ciertos valores que consideren relevantes para sus propias prácticas.

El nivel micro hace referencia a cómo un matemático en particular hace matemáticas y cuáles son sus creencias al respecto de éstas, las cuales pueden no estar alineadas con las de la comunidad a la que éste pertenece. En este trabajo en ningún momento hablaremos de este nivel, pues no es relevante para nuestros propósitos. ${ }^{5}$

En la sección 2 mostraremos la importancia que esta distinción tiene para algunas asunciones básicas de la filosofía de las prácticas matemáticas, específicamente, aquellas que enfatizan la importancia de la historia para el análisis del conocimiento matemático, así como el papel que desempeñan los agentes que conforman las comunidades matemáticas en su desarrollo. En esta misma sección presentaremos una propuesta particular, dentro de este marco general, en la que se considerarán los diagramas como herramientas manipulativas (Giardino 2013). Posteriormente, aplicaremos dicho marco teórico en nuestro caso de estudio, esto es, en los Elementos de Euclides (sección 3) así como en el Zhou bi y en los Nueve capítulos (sección 4), haciendo énfasis en el uso de diagramas para la demostración del teorema de Pitágoras o procedimiento gou gu. Por último, en la sección 5 presentaremos las similitudes y diferencias que podemos encontrar entre estas dos prácticas matemáticas. Destacaremos la idea de que, aunque cada una de estas prácticas siguiera estrategias diferentes a la hora de desarrollar el conocimiento matemático, ambas estarían guiadas por dos valores fundamentales, como la generalidad y la exactitud de sus resultados. Por último, discutiremos algunas posibles consecuencias de nuestro análisis contextual para la evaluación de la mayor o menor "matematicidad" de las distintas prácticas analizadas, así como

${ }^{5}$ Por ejemplo, Ferreirós presenta las razones que Weyl adujo para afirmar que los números reales clásicos no formaban un dominio cerrado (2016, pp. 203-204). Esto es, Weyl — nivel micro - rechaza ciertos axiomas — digamos estándares para la comunidad matemática - basándose en sus creencias —intuicionistas - de cómo deben ser las matemáticas. 
la posible valoración de su importancia dentro de la historia de las matemáticas.

\section{Sobre prácticas matemáticas y diagramas}

En las últimas décadas un mayor número de investigadores se ha interesado en el análisis de las prácticas matemáticas, como puede verse en el libro que Mancosu (2008) edita, o en las monografías recientes de Ferreirós (2016) o Wagner (2017). Este interés viene unido a la asunción de que para analizar y entender apropiadamente la emergencia y evolución del conocimiento matemático tenemos que centrar nuestro interés en cómo los matemáticos hacen matemáticas.

En este sentido, cada comunidad matemática — nivel meso - desarrollará diferentes herramientas dentro de sus prácticas, se centrará en problemas determinados, elaborará diferentes consideraciones teóricas según las metas y valores por los que se guíen, etc., todo ello relacionado con el contexto en el que dichas prácticas emerjan y evolucionen. ${ }^{6}$ Por lo tanto, la actividad matemática no se considera algo estático, sino un fenómeno complejo donde diferentes aspectos emergen conjunta e interactivamente, influenciados por ciertos condicionantes - teóricos, metodológicos, etc. — de cada comunidad matemática en cada contexto (Ferreirós 2016).

En este trabajo centraremos nuestra atención en un tipo particular de herramientas, los diagramas, cuyas principales características presentaremos a continuación. Como mostraremos en la sección 5, cada comunidad matemática hará un uso diferente de estas herramientas, y les otorgará un determinado estatus epistemológico. Esto, por supuesto, forma parte de la naturaleza histórica y contextual de las matemáticas que defendemos en este trabajo.

Autores como Manders (2008a; 2008b), Giardino (2013) y Ferreirós (2016) han reevaluado el valor epistemológico de los diagramas en las prácticas matemáticas, así como su importancia en la justificación, explicación y/o entendimiento matemático; ya sea en el uso de diagramas en general, como lo hace Giardino, o con especial atención a la práctica euclidiana, como lo hacen Ferreirós y Manders.

Nuestra propuesta se centra en la idea de que los diagramas son herramientas, y que precisamente por la posibilidad de manipularlos podemos justificar su valor epistemológico dentro de las diferentes prácticas matemáticas (Giardino 2013). De manera general, podemos

${ }^{6}$ Véase De Regt y Dieks (2005, p. 161) para una presentación general de esta aproximación contextual aplicada a las prácticas científicas y el uso de diferentes herramientas. 
entender diagrama como "todos los casos de representaciones en dos dimensiones, siendo esta bidimensionalidad relevante para la manera en la que la información es presentada y leída en ellos" (Giardino 2017, p. 500). Por lo tanto, el énfasis se pone en los diagramas como herramientas cognitivas que permiten mostrar información espacialmente, que el matemático usa para diferentes tareas en su propia práctica matemática (Giardino 2017).

En esta propuesta, los diagramas se analizan en relación con los agentes que los crean y utilizan. De esta manera, lo importante es entender las razones por las que se diseñó y para qué va a ser utilizado, esto es, la intención detrás de su construcción. Así mismo, se puede afirmar que los diagramas no poseen contenido de manera independiente a un sistema particular de representación — conjunto de reglas implícitas o explícitas de construcción e interpretación de éstos-, sino que para poder extraerles contenido con sentido y reconocer sus funciones, el agente debe aprender a leerlos e interpretarlos. Sólo cuando un agente aprende a construir e interpretar los diagramas de acuerdo con una práctica específica se vuelve competente en su manipulación y en la extracción de resultados de manera segura y útil. Aprender a usar un diagrama implica, por lo tanto, aprender a construirlo y aprender sus reglas interpretativas, las cuales, nos permiten ser competentes en la interpretación de varias construcciones dentro del mismo sistema (Giardino y Casati 2013; Giardino y Greenberg 2014).

Por lo tanto, manipular e interpretar un diagrama dentro de una práctica particular no consiste meramente en la percepción de sus aspectos estáticos, sino también en la consideración de las acciones que podemos llevar a cabo en ellos y con ellos, e identificar los posibles resultados que emerjan. En esta práctica, diagramas y textos trabajan cooperativamente para su correcta construcción e interpretación, y el diagrama no es una mera traducción visual del texto. La geómetra, por así decirlo, construye y razona con el diagrama sin considerar cómo está dado visualmente, sino por cómo ésta lo concibe, pudiendo afirmar así que "el diagrama se convierte en el lugar de trabajo del matemático, donde las operaciones, planes y experimentos se llevan a cabo para encontrar soluciones y razones para éstas" (Giardino 2013, p. 146).

Como ejemplo ilustrativo de este hecho considérese el siguiente diagrama (Figura 1, p. 11), en el que estamos viendo la línea AB tangente a un círculo, y el punto $\mathrm{E}$ marcando el único punto que línea y círculo comparten en común. Así se pone de manifiesto que lo importante no es cómo percibimos visualmente el diagrama, puesto que 
sólo fijándonos en sus características visuo-espaciales no podríamos afirmar que comparten un solo punto. Lo importante, dentro de esta caracterización del razonamiento diagramático, es cómo los agentes, de acuerdo con una práctica matemática compartida — en este caso, la práctica euclidiana-, manipulan este diagrama y la información que pueden extraer gracias a esa cooperación diagrama-texto. En este caso en particular es la información textual - la definición de recta tangente a una curva - la que nos permite saber que la línea es tangente al círculo dado, y no la información diagramática.

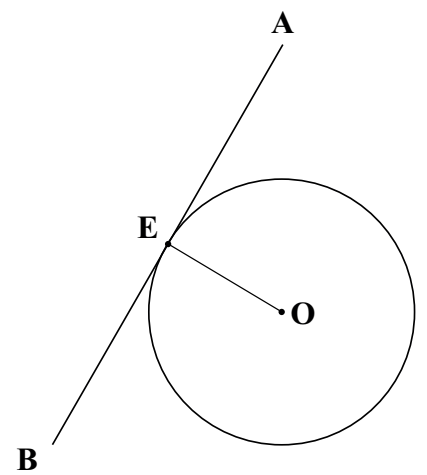

Figura 1. Diagrama de la recta $\mathrm{AB}$ tangente al círculo.

Una vez presentado este marco teórico general, analizaremos el desarrollo de conocimientos, así como la presentación de los resultados matemáticos en las dos prácticas matemáticas de la antigüedad mencionadas anteriormente.

\section{Práctica matemática y uso de diagramas en los Elementos}

Los Elementos de Euclides se escribieron en algún momento alrededor del 300 a.e.c. Algunos historiadores la consideran la obra que inauguró el curso - deductivo - que tomaría lo que denominamos matemáticas. ${ }^{7}$ En esta sección nos centraremos en los diagramas que Euclides hace en los Elementos, con énfasis en la demostración del teorema de Pitágoras, sin entrar en detalles acerca de la historia de esta obra. ${ }^{8}$

${ }^{7}$ Para una presentación general y crítica de esta interpretación, véase el primer capítulo del libro de Joseph (2011).

${ }^{8}$ Heath (1921; 1956) y Artmann (1999), entre otros, cubren bien esta parte. 
Existe una extensa literatura sobre el uso de diagramas en la geometría de Euclides, y Giardino (2017) presenta una revisión general de ellos. Subrayaremos tres características importantes de los diagramas en esta práctica matemática: 1) uso de letras, que conectan diagrama y texto (Netz 1999); 2) la conjunción de información exacta y co-exacta (Manders 2008a; 2008b); y 3) consideración de las definiciones y postulados como reglas de construcción e interpretación de los diagramas (Ferreirós 2016).

La primera característica se refiere a la combinación de letras - en el texto- y propiedades visuo-espaciales —en el diagrama-, procedimiento por el cual se conecta precisamente texto y diagrama. $\mathrm{Su}$ uso sigue algunas convenciones implícitas en los Elementos, que son necesarias además para comprender su construcción y para razonar de manera correcta con éstos. Netz (1999, pp. 68-83) denomina "bautismo" a esta práctica de conectar letras individuales con objetos individuales, y cuando diversos elementos están bautizados, pueden formarse nombres con varias letras. Generalmente las letras individuales representan puntos, y los nombres con varias letras representan objetos mediante sus puntos de conexión. Por lo tanto, un diagrama estaría conectado por un conjunto de puntos, y recibiría su nombre a través de ellos. Por ejemplo, A y B pueden representar dos puntos, pero estos puntos pueden ser los extremos de una línea recta, que será la línea $\mathrm{AB}$.

Esta práctica de bautismo sigue, por lo general, una secuencia alfabética, y la repetición de cualquier letra o conjunto de éstas designaría al mismo objeto a lo largo de la prueba. Además, pueden existir cambios de nombres, como que una línea $A B$ sea designada $\mathrm{BA}$, los cuales seguirían también un orden lineal como los bautismos, y estarían sujetos a ciertas restricciones que Netz (1999) presenta.

En segundo lugar, Manders (2008a; 2008b) propone la distinción entre información exacta y co-exacta. Información exacta es la información que podemos extraer del texto, en la que se incluye la igualdad de segmentos, ángulos y otras magnitudes, relaciones de congruencia y proporcionalidades. Por el contrario, la información co-exacta se extrae del diagrama, aspectos diagramáticos que permanecen estables bajo pequeñas deformaciones o mejoras en el dibujo, e incluyen, entre otras, relaciones parte-todo de regiones y puntos de intersección. Por ejemplo, en I.1 - proposición acerca de cómo construir un triángulo equilátero - tenemos la siguiente información exacta: "siendo el punto A el centro del círculo CDB, AC es igual a $A B$ ". Ésta es una información - un punto siendo el centro de 
un círculo - que sólo puede saberse por lo que diga el texto, y no es estable bajo deformaciones del dibujo. La información co-exacta sería: "desde el punto $\mathrm{C}$, en el cual los círculos se cortan el uno al otro". Este punto de intersección sería parte de los aspectos coexactos del diagrama, el cual aparecerá aunque dibujemos mal los círculos, deformados, como podemos ver en la Figura 2.

Según Manders (2008a; 2008b), dos características importantes de la construcción de diagramas son las siguientes. En primer lugar, el diagrama está sujeto a las atribuciones exactas, esto es, si la información exacta dice que dibujemos una línea recta, sabemos que ésta es recta aunque en el diagrama no la dibujemos siendo perfectamente recta. Pero, no todas las deformaciones diagramáticas están permitidas. Una construcción con líneas exageradamente curvas no se admitirá pues podría interferir en los aspectos co-exactos. En segundo lugar, tenemos que considerar la disciplina diagramática, que es la habilidad para producir diagramas suficientemente buenos y controlar su apariencia, reconociendo así los aspectos co-exactos que aparecerán en el diagrama. Esta habilidad, de acuerdo con Manders, es aprendida dentro de la propia práctica griega a través de la presentación, inspección, y sustitución de dibujos malos cuando sea el caso $(2008 \mathrm{~b}) .^{9}$
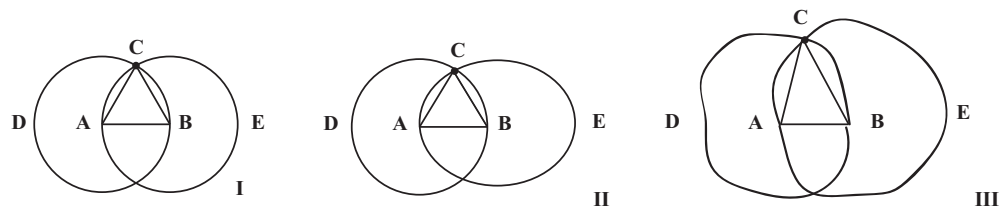

Figura 2. En estos tres diagramas podemos ver que la información coexacta, el hecho de que A sea interior al círculo, o que aparezca el punto de intersección C, es estable bajo deformación del propio diagrama.

En la práctica demostrativa euclidiana lo primero es enunciar una proposición, ejemplificada por un diagrama concreto, y entonces se especifica qué es lo que se va a probar. La prueba comienza con construcciones permitidas por los postulados, o por proposiciones probadas anteriormente - teoremas o problemas-, y en los argumentos

${ }^{9}$ Un hecho histórico a favor de esta interpretación es la existencia de un trabajo de Euclides titulado Pseudry o Pseudographemata, el cual conocemos sólo por fuentes secundarias, como Proclo (quien lo llamaba el Libro de las falacias). Este libro podría haber tenido la intención de llamar la atención del geómetra para evitar falacias basadas en las apariencias de los diagramas (Heath 1921, pp. 430-431). 
demostrativos se establece la validez de lo afirmado, basándose en las definiciones y nociones comunes. Aquí es donde hay una división de trabajo entre la información exacta y co-exacta, y esta última consiste en elementos que se introducen en el diagrama siguiendo lo que se estipula textualmente más las regiones, segmentos y puntos que pueden emerger - como se mostró en I.1 anteriormente, y como explicaremos más detalladamente en el caso de I.47, el teorema de Pitágoras-. Así, de acuerdo con Manders (2008b), cada paso en las pruebas euclidianas "consiste en una atribución en el texto discursivo, o en una construcción en el diagrama, o en ambos" (p. 87).

En tercer lugar, Ferreirós (2016, pp. 112-152) propone una interpretación del papel de las definiciones en la geometría de Euclides, y considera que las definiciones se pueden entender como elementos utilizados para enseñar a un aprendiz a leer los diagramas, razonar con ellos, y entender qué implican. Así, uno sabe reconocer un punto en el dibujo, no por poder representarlo de una manera precisa - lo cual es imposible, pues punto según la definición primera es aquello que no tiene partes-, sino más bien porque comprende la definición y sabe interpretar el dibujo de acuerdo con ella dentro de la práctica euclidiana.

Además, los postulados constriñen y controlan la construcción de los diagramas. Los tres primeros postulados indican construcciones o acciones que pueden realizarse en el diagrama (aitemata, que significa literalmente "cosas que son requeridas"). Como observa Ferreirós:

Proclo está llamando nuestra atención sobre el hecho de que los postulados tienen una naturaleza proactiva y pragmática: ellos permiten al geómetra hacer ciertas cosas, operar con diagramas de ciertas maneras, y fuerzan a un posible oponente a aceptar ciertos movimientos diagramáticos. Esto debe ser claro y obvio para los tres primeros postulados, los cuales aparecen como reglas procedurales que regulan la construcción de los diagramas (2016, p. 128).

El primer postulado nos permite realizar en el diagrama la acción de dibujar una línea recta entre dos puntos dados, el segundo extender una línea recta indefinidamente, y el tercero dibujar un círculo desde un punto y con un radio dado. Estas reglas de construcción estarían constriñendo las operaciones que la geómetra puede llevar a cabo en el diagrama, permitiendo a un posible interlocutor (maestro o alumno, por ejemplo) aceptar o no los movimientos diagramáticos y la información que de ellos se infiere. 
Entonces, aprender a construir un diagrama adecuado implica saber cómo manipularlo de acuerdo con las prescripciones textuales, así como reconocer los aspectos relevantes que emergen de dichas manipulaciones. En relación con esto, Ferreirós afirma: "la geómetra actuará (dibujando) y razonará (infiriendo) consecuentemente, empleando el diagrama no como es dado empíricamente, sino como es concebido" (2016, p. 144).

Por consiguiente, los agentes que comparten esta práctica poseen recursos para controlar la construcción e interpretación de los diagramas, mejorar su fiabilidad, y permitir resolver desacuerdos entre interpretaciones alternativas basadas en la apariencia del dibujo. Aseguran, por lo tanto, el uso de las atribuciones co-exactas como base para las inferencias.

El análisis de los autores que hemos presentado ha sido muy influyente y ha aclarado la interrelación entre texto y diagrama en las pruebas presentes en los Elementos. Netz esclarece las normas implícitas en el bautismo del diagrama a partir de las letras y de cómo el texto se refiere a los diagramas a lo largo de los Elementos. Manders presenta la metodología estándar de las pruebas euclidianas, dividida entre construcción y demostración de un problema o teorema, y aclara cuál es la función de la parte textual y diagramática en las pruebas. Aunque no presentemos en detalle este punto, uno de los méritos de este análisis es enseñar que el uso de diagramas es uniforme tanto en las pruebas directas como en las pruebas por reductio, y explica que el establecimiento de la contradicción en estas últimas se da en general por la división del trabajo entre información exacta y co-exacta. ${ }^{10}$ Estos análisis muestran que el uso de los diagramas en Euclides es uniforme, que está regimentado por los postulados en la construcción, y que es fiable al emplearse en el argumento con respecto a sus aspectos co-exactos. ${ }^{11}$

Aunque la estructura que presentamos sea representativa de la mayoría de las pruebas de los Elementos, nos limitaremos a analizar el teorema de Pitágoras - I.47 en los Elementos - tal y como lo demuestra Euclides. Elegimos este ejemplo por dos razones. En

\footnotetext{
${ }^{10}$ Véase un ejemplo de ello en Manders (2008b, pp. 103-109), donde el autor presenta un análisis de la proposición I.6.

${ }^{11}$ El análisis de Manders sirvió como base para algunas formalizaciones de los Elementos, como la que Avigad, Mumma y Dean (2009) presentan. Esta formalización muestra el éxito del análisis del autor con respecto a la división del trabajo entre la información exacta y co-exacta. En el sistema de Avigad et al., solamente los aspectos co-exactos son formalizados, manteniéndose fiel con respecto a la estructura de las pruebas de los Elementos.
} 
primer lugar, por la posibilidad de comparar este resultado con el de la antigua civilización china; y en segundo lugar, puesto que en esta prueba quedan ejemplificadas las características de la práctica euclidiana presentadas anteriormente.

En I.47 (Figura 3, pp. 16-17), Euclides prueba que para cualquier triángulo rectángulo, el cuadrado del lado opuesto al ángulo recto es igual a los cuadrados de los lados que contienen a dicho ángulo recto. La manera en que Euclides lo prueba es compleja, puesto que involucra diferentes elementos como construcciones en el diagrama, nociones comunes, y varios resultados probados anteriormente como I.14, I.31, I.41 o I.46. ${ }^{12}$ Nos parece relevante destacar, dada la larga discusión histórica que hubo en torno al postulado $\mathrm{V}$, que algunas de estas proposiciones, como la I.41 y I.46, dependen de I.29, la primera proposición en los Elementos en la que Euclides hace uso de dicho postulado.

\section{Proposición 47}

En los triángulos rectángulos el cuadrado del lado que subtiende el ángulo recto es igual a los cuadrados de los lados que comprenden el ángulo recto.

Sea $\mathrm{ABC}$ el triángulo rectángulo que tiene el ángulo recto BAC.

Digo que el cuadrado de BC es igual a los cuadrados de BA, AC.

Trácese a partir de BC el cuadrado BDEC, y a partir de BA, AC los cuadrados BG, HC [I.46], y por el (punto) A trácese AL paralela a una de las dos (rectas) $\mathrm{BD}, \mathrm{CE}$; y trácese $\mathrm{AD}, \mathrm{FC}$. Y dado que cada uno de los ángulos $\mathrm{BAC}, \mathrm{BAG}$ es recto, entonces en una recta cualquiera $\mathrm{BA}$ y por un punto de ella, $\mathrm{A}$, las dos rectas AC, AG, no colocadas en el mismo lado, hacen los ángulos adyacentes iguales a dos rectos; por tanto, CA está en línea recta con AG [I.14]. Por la misma razón, BA está en línea recta con AH. Y como el ángulo DBC es igual al (ángulo) FBA - porque cada uno (de ellos) es recto - añádese a ambos el (ángulo) ABC; entonces el (ángulo) entero DBA es igual al (ángulo) entero FBC [N.C. 2]; y como DB es igual a BC, y FB a BA, los dos (lados) DB, BA son iguales respectivamente a los dos (lados) FB, $\mathrm{BC}$; y el ángulo DBA es igual al ángulo FBC; entonces la base $\mathrm{AD}$ es igual FC, y el triángulo $\mathrm{ABD}$ es igual al triángulo FBC [I.4]; y el paralelogramo BL es el doble del triángulo $\mathrm{ABD}$ : porque tienen la misma base $\mathrm{BD}$ y están entre las mismas paralelas BD, AL [I.41]; pero el cuadrado GB es el doble del triángulo FBC: porque tienen

${ }^{12}$ En I.46 se demuestra que, sobre una recta dada, se puede construir un cuadrado. En I.4l que si un paralelogramo y un triángulo tienen la misma base, y están entre las mismas paralelas, la superficie del paralelogramo es el doble que la superficie del triángulo. En I.14 se prueba que en cualquier recta y a partir de uno de sus puntos, si se levantan dos rectas que no están del mismo lado y forman ángulos adyacentes iguales a dos rectos, entonces las dos rectas están en línea recta una con la otra. Con I.3l se permite que por un punto se pueda trazar una línea recta paralela a una recta dada. Por otro lado, también se hace uso de I.4, que es conocido como el teorema de congruencia de triángulos lado-ángulo-lado. 
a su vez la misma base FB y están entre las mismas paralelas FB, GC [I.41]; [pero los dobles de cosas iguales son iguales entre síl; por tanto, el paralelogramo BL es también igual al cuadrado GB. De manera semejante, trazando las (rectas) AE, BK se demostraría también que el paralelogramo CL es igual al cuadrado HC; por tanto, el cuadrado entero BDEC es igual a los cuadrados GB, HC [N.C. 2]. Asimismo, el cuadrado BDEC ha sido trazado a partir de BC, y los (cuadrados) GB, HC a partir de BA, AC. Por tanto, el cuadrado del lado BC es igual a los cuadrados de los lados BA, AC.

Por consiguiente, en los triángulos rectángulos el cuadrado del lado que subtiende el ángulo recto es igual a los cuadrados de los lados que comprenden el ángulo recto. Q.E.D.

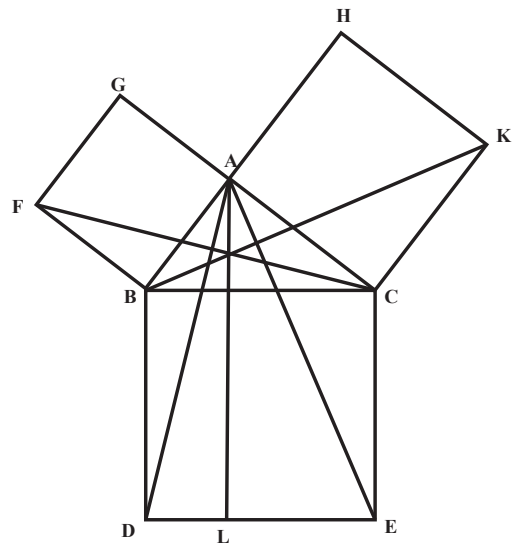

Figura 3. Prueba del teorema de Pitágoras por Euclides en I.47, siguiendo la traducción de Puertas Castaños (2000, pp. 79-80).

En este caso podemos ver todas las características anteriormente explicadas de cómo se usan los diagramas en Euclides: 1) texto y diagrama han de ser considerados en conjunto, estando ambos conectados por el uso de las letras; 2) que "BAC es un ángulo recto" es parte de la información exacta, pues ésta se deriva de la información textual de que éstos son los ángulos de un cuadrado, y que los ángulos de los cuadrados son rectos; 3) que i) el ángulo CBA más el FBA forman el FBC, y ii) que el ángulo CBA más el CBD forman el ABD, es información co-exacta que se extrae directamente del diagrama; 4) que los ángulos ABD y FBC son iguales se justifica no por el diagrama sino por la información del texto, esto es, exacta; 5) la construcción tanto del primer triángulo como de los cuadrados es permitida por teoremas que han sido probados anteriormente; y 6) la construcción 
de las líneas, tales como "AD" o "FC", se hace de acuerdo con los postulados, y particularmente de acuerdo con el postulado primero. Véase la Figura 3 donde está desarrollada la prueba completa.

En esta sección hemos querido mostrar las principales características de la práctica diagramática en la geometría de Euclides tal y como diversos autores las han analizado y presentado. En nuestro caso de estudio, nos hemos centrado en el análisis de la demostración del teorema de Pitágoras, en I.47, viendo cómo se cumplen de manera general las asunciones teóricas presentadas anteriormente.

\section{Práctica matemática y uso de diagramas en el Zhou bi y en los Nueve capítulos}

$\mathrm{Al}$ contrario de lo que ocurre con la matemática griega, no existe una literatura extensa acerca de esta tradición matemática. ${ }^{13}$ Por ello, en esta sección introduciremos una breve presentación de dos de los primeros y principales libros de astronomía y matemáticas de esta civilización, en los cuales enfocaremos nuestro análisis acerca del uso de diagramas en el procedimiento gou gu.

Centraremos nuestra atención en el Zhou bi y en los Nueve capítulos, particularmente en los comentarios realizados en el III e.c. por Zhao Shuang y Liu Hui, respectivamente. Como ocurre con los Elementos, es difícil saber con exactitud la fecha de compilación de estas obras. Algunos autores (Cullen 1996; Chemla y Guo 2004) creen que éstos fueron compilados entre el siglo I a.e.c y el I e.c., aunque existe una discusión amplia acerca de ello. Como los Elementos, los textos originales no han sobrevivido hasta nuestra época, ni siquiera las versiones con los comentarios del siglo III e.c.

Estas obras pasaron rápidamente a formar parte del canon matemático de esta civilización, convirtiéndose en el primer y segundo capítulo (respectivamente) de los Diez clásicos de las matemáticas (Suanjing shishu), obra compilada por primera vez en el siglo VII e.c. (Cullen 1996, p. 74; Martzloff 1997, pp. 123-126).

El Zhou bi puede considerarse un libro astronómico, posiblemente previo a los Nueve capítulos. En relación con su contenido matemático, hay que decir que todo el material que aparece en este libro - excepto por un principio conocido como "principio de la

\footnotetext{
${ }^{13}$ Hay algunos libros que han tratado de dar cuenta de esta historia, como Martzloff (versión francesa publicada en 1988, e inglesa en 1997). Así mismo, autores como Cullen (1996), Chemla y Guo (2004), y Dauben (2008) han traducido y comentado algunos de los libros más importantes de esta civilización a lenguas occidentales.
} 
sombra" - se encuentra mejor ejemplificado, y presentado de manera más sistemática, en los Nueve capítulos (Cullen 1996, p. 74).

El Zhou bi, por un lado, no posee una estructura común en la presentación de procedimientos y resultados astronómicos y matemáticos, mientras que en los Nueve capítulos se mantiene una misma estructura durante toda la obra. Dicha estructura consiste, en primer lugar, en presentar el problema que se quiere resolver, a continuación se da la solución, y finalmente se muestra el procedimiento ${ }^{14}$ que habría que seguir para resolver el problema. Así mismo, como Cullen (1996) comenta, hay secciones del Zhou bi que se introducen sin tener una conexión clara con las demás, como es el caso de la sección \#C, la cual consiste en dos párrafos donde se incluyen procedimientos acerca de las relaciones entre círculo y cuadrado (pp. 181-182). En los Nueve capítulos hay una ordenación clara, cada capítulo está dedicado a mostrar un conjunto de problemas del mismo tipo a resolver con uno o varios procedimientos generales. Por ejemplo, el capítulo nueve trata problemas relacionados con lo que occidentalmente denominamos triángulos rectángulos, ${ }^{15}$ y algunos procedimientos generales para resolver dichos problemas.

Se cree que en las obras originales no aparecían diagramas, y que fueron precisamente los primeros comentaristas los que los introdujeron en sus comentarios. Tanto Zhao Shuang (Cullen 1996, p. 171) como Liu Hui (Chemla y Guo 2004, p. 127) afirman en la introducción a sus comentarios que las razones principales para su uso fueron pedagógicas, y explicativas.

Ambos comentaristas usaron estos diagramas con el fin pedagógico de hacer sus contenidos más sencillos para futuros alumnos, así como para otros matemáticos interesados en su estudio. En cuanto al papel explicativo, se cree que con estos diagramas los comentaristas intentaron resolver el vacío explicativo de las obras originales, en las que simplemente se presentaba el procedimiento para resolver los

${ }^{14}$ Chemla y Guo (2004, pp. 21-26) señalan que usan indistintamente "procedimiento" y "algoritmo" en su traducción y comentarios a los Nueve capítulos. Se entiende algoritmo como "listas de operaciones", esto es, series finitas de operaciones que son ejecutadas en un orden determinado, que comparten los valores dados, y producen así mismo el valor deseado (p. 21).

${ }^{15}$ Cullen (1996, pp. 77-80) señala que en la matemática china antigua nunca se usa el término de triángulo al estilo griego de trigonom. En estas dos compilaciones sólo se habla acerca de las relaciones entre un "gancho horizontal" - lado pequeño, gou - una "pierna" - lado largo, gu-, y uniendo sus finales una "cuerda de arco" -hipotenusa, xian - Tampoco se usa el concepto de ángulo en sentido euclidiano, pues el énfasis, como hemos dicho, no está en los ángulos que estas líneas forman, sino en sus valores y ratios (Cullen 1996, p. 92). 
problemas, y a veces era difícil saber por qué ese procedimiento era correcto. En este sentido, Volkov usa el término "diagramas conceptuales", ya que "su función principal era la de proveer descripciones de las transformaciones geométricas a realizar para justificar los algoritmos matemáticos" (2007, p. 457).

En la tradición china se usaron dos términos para hablar sobre diagramas, $t u$ - figuras - y $q i^{16}$ — bloques - (Chemla y Guo 2004; Chemla 2010). El primero lo usaron tanto Zhao Shuang como Liu Hui para resolver problemas de geometría plana, mientras que el segundo sólo lo utilizó Liu Hui para tratar la geometría del espacio.

Estos diagramas, al igual que los de los Elementos, no los utilizan como meras ilustraciones estáticas de una forma particular en relación con un problema dado. Como afirma Chemla (2010), los diagramas "son usados para hacer explícito el "significado $y i$ 意" de operaciones" (p. 314, nota 21). Esto es, el papel explicativo y pedagógico que fue mencionamos anteriormente.

Al hacer uso de estos diagramas, los comentaristas usan expresiones que hacen referencia explícita a su manipulación, tales como "cortar" las figuras, "unirlas", "romper los rectángulos", "colorear", etc. (Chemla 2010). Este hecho, entre otros que Chemla (2010) presenta, lo han llevado a la hipótesis de que estos diagramas fueron objetos físicos que los matemáticos manipularon al mismo tiempo que llevaban a cabo los procedimientos. Autores como Volkov (2007) presentan una hipótesis alternativa, y afirman que existieron libros que fueron publicados por separado de los tratados clásicos de matemáticas en los que sólo aparecían los diagramas (pp. 432-434). Una característica de estos diagramas es que, a diferencia de los que aparecen en los Elementos, no poseían letras para conectar texto y diagrama, sino que esta conexión se hacía mediante el uso de colores (véase la siguiente sección).

Aunque sería interesante analizar las diferencias entre el uso de diagramas $t u$ y diagramas $q i$ en los Nueve capítulos, éste es un tema que se aleja del interés principal de nuestro trabajo. Nos centraremos en analizar el uso de diagramas $t u$, puesto que éstos son los que aparecen en relación con el procedimiento gou gu. Y lo interesante para nuestra presentación es que, ya fueran diagramas griegos pintados en tierra o cenizas (Netz 1999, pp. 14-16), o diagramas $t u$ pintados en

\footnotetext{
${ }^{16}$ Según Chemla y Guo, en el glosario de términos encontramos que $t u$ se define como "el asistente de razonamiento, instrumento de visualización, para la geometría plana" (2004, p. 999); mientras que qi es definido como "el asistente de razonamiento, el instrumento de visualización para la geometría del espacio" (2004, p. 967).
} 
papel, el aspecto relevante de ambos es que fueron representaciones en dos dimensiones usadas como herramientas manipulativas en cada práctica matemática. A continuación presentaremos sus principales características en relación con el procedimiento gou gu.

El diagrama relacionado con el procedimiento gou gu aparece por primera $\mathrm{vez}^{17}$ en la tradición china en la sección \#A del Zhou $b i$, específicamente, en los comentarios de Zhao Shuang a dicha obra. Por lo tanto, este procedimiento nace en esta civilización en el contexto de la astronomía y la topografía (Cullen 1996, p. 87). Zhao Shuang, sin embargo, sólo usará la relación entre gou y gu, mientras que se menciona a xian (hipotenusa), pero no forma parte esencial del procedimiento en esta obra (Chemla y Guo 2004, p. 662).

Veamos a continuación la traducción de Cullen (1996) al texto "Diagramas de base y altura, círculos y cuadrado", que añade Zhao Shuang al libro \#A del Zhou bi (p. 206). En primer lugar, Zhao Shuang muestra cuál es el procedimiento, "La base y la altura están cada una multiplicadas por sí mismas. Añádelas para hacer el área de la hipotenusa. Divídelo para abrir el cuadrado, ${ }^{18}$ y ésta es la hipotenusa" (Cullen 1996, p. 208).

Éste sería el procedimiento gou gu, al cual Zhao Shuang añade posteriormente el diagrama de la hipotenusa (Figura 4, p. 22) para explicar por qué este procedimiento es correcto, y hacerlo entendible a matemáticos o lectores de esta obra,

de acuerdo con el diagrama de la hipotenusa, debes multiplicar la base y altura juntos para hacer dos de las áreas rojas. Dóblalo para hacer cuatro de las áreas rojas. Multiplica la diferencia de la base y la altura por ellos mismos para hacer el área central amarilla. Si se añade [tal] diferencia [a las cuatro áreas rojas], se completa el área de la hipotenusa (Cullen 1996, p. 208).

Como dijimos anteriormente, la obra de los Nueve capítulos es más interesante en cuanto a contenido matemático que el Zhou bi, y presenta de una manera más sistemática el contenido de la misma. En este texto, hay un capítulo completo - capítulo 9- dedicado al

${ }^{17} \mathrm{El}$ Suan shu shu - Libro sobre números y computación- (Dauben 2008) es una obra del siglo II a.e.c., anterior a la sección \#A del Zhou bi, en la que no aparece el procedimiento gou gu debido a que éste era un tratado sobre problemas administrativos y financieros para el que no era necesario desarrollar tal procedimiento (Chemla y Guo 2004, p. 5).

${ }^{18}$ Esta expresión, según Cullen (p. 208, nota 226) es la expresión que se utiliza en china para hacer referencia a la extracción de raíces. 
procedimiento gou gu, donde se usan cuatro figuras fundamentales, reducibles a dos, ${ }^{19}$ para resolver los problemas del 9.1 al 9.12, y el 9.24. Los problemas del 9.13 al 9.23 se resuelven por lo que conocemos como regla de tres - lü- (Chemla y Guo 2004, p. 673).

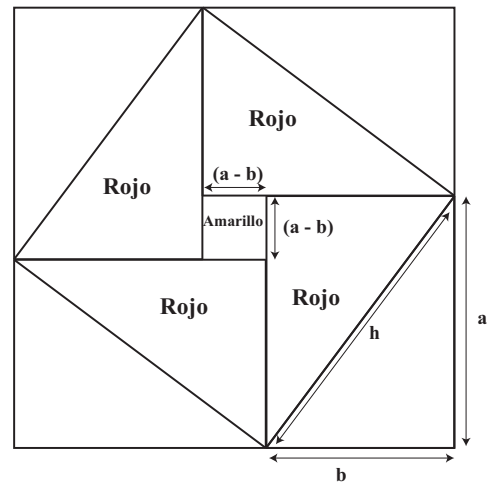

Figura 4. Reconstrucción del diagrama Xian presentada por Cullen (1996) que acompañaría los comentarios de Zhao Shuang a la sección \#A del Zhou bi (p. 209).

Veamos ahora la traducción de Chemla y Guo (2004) del problema 9.1 tal y como Liu Hui lo presenta,

(9.1) Supongamos que la base (gou) sea de $3 c h i^{20}$ y la altura $(g u)$ de 4 chi. Se pide cuánto hace la hipotenusa.

Respuesta: 5 chi.

Procedimiento de la base (gou) y de la altura $(g u)$ : siendo base (gou) y altura ( $g u)$ cada uno multiplicados por sí mismos, se suman (los resultados) y esto se divide por la extracción de la raíz cuadrada, lo que nos da la hipotenusa (p. 705). ${ }^{21}$

Esta estructura, de primero presentar el problema, dar la solución y, por último, exponer el procedimiento utilizado para alcanzar dicha

${ }^{19}$ No es nuestro interés en este trabajo analizar cuáles son estas dos figuras fundamentales. Para más información, véase Chemla y Guo 2004 (pp. 673-684).

${ }^{20}$ Chi es una unidad de medida utilizada en la antigua civilización china que equivale a unos 23-24 cm (Chemla y Guo 2004).

${ }^{21}$ Hemos modificado ligeramente la exposición de Chemla y Guo (2004, p. 705) del problema 9.1, pues éste viene junto al 9.2 y 9.3, los cuales se resuelven con el mismo procedimiento, y hacen uso del mismo diagrama. 
solución, es la que siguen los 246 problemas que componen los Nueve capítulos.

El diagrama (Figura 5) que acompaña a este procedimiento, según la reconstrucción de Chemla y Guo (2004, p. 879), Liu Hui lo utilizó con la intención de mostrar que el algoritmo presentado en la obra original de los Nueve capítulos es correcto.

En el caso particular de 9.1 podemos ver que si multiplicamos la base por ella misma obtendremos el cuadrado bermellón, y al multiplicar la altura por sí misma obtendríamos el cuadrado azulverde (Figura 5A). Entonces, realizando movimientos rígidos como podemos ver desde $5 \mathrm{~A}$ a $5 \mathrm{~B}$, generamos el cuadrado del lado de la hipotenusa, llegando a la solución que ya se había proporcionado.

Como podemos observar, en estas obras los diagramas se utilizan como herramientas manipulativas con los que se persiguen ciertos objetivos. En este caso, tal y como los mismos comentaristas señalan, su intención al usarlos es mostrar por qué los procedimientos que encontramos en los clásicos matemáticos de la antigua civilización china eran correctos, y de esta manera poder hacerlos más accesibles al público que los estudiara. Además, estos diagramas no son una mera traducción visual de estos procedimientos, sino que procedimientos y diagramas forman un todo unido, el cual, es parte esencial de esta práctica matemática.

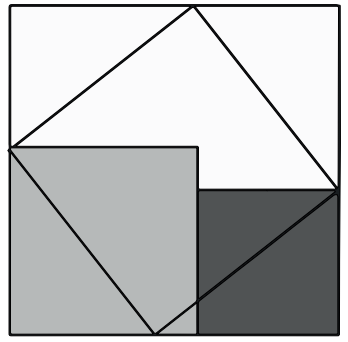

Bermellón Azul-verde

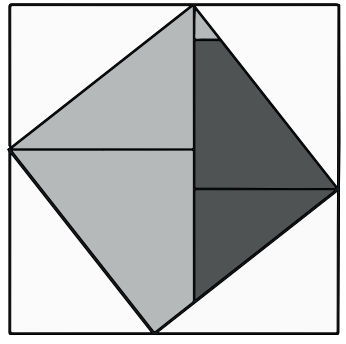

Figura 5. Reconstrucción de Chemla y Guo (2004, p. 879) del diagrama que acompaña al procedimiento gou gu en los problemas 9.1, 9.2 y 9.3 de los Nueve capítulos, denominado como la primera figura fundamental. 


\section{Análisis comparativo del uso de diagramas}

En esta sección vamos a analizar las diferencias y similitudes que existen en el uso de diagramas en los textos presentados anteriormente, y veremos de qué manera nuestro marco teórico sirve para entender estos desarrollos matemáticos de la antigüedad.

Existen numerosas diferencias entre cómo estos diagramas se utilizaron en estas prácticas matemáticas, las cuales pueden agruparse en dos categorías generales. En primer lugar, frente a la estrategia deductiva de los Elementos encontramos en las obras chinas una estrategia heurística de búsqueda de la generalidad de los procedimientos. En segundo lugar, la importancia de los movimientos en la matemática china frente a su poca relevancia, y casi ausencia, en los Elementos. ${ }^{22}$

Respecto a la primera categoría, podemos decir que en el libro I de los Elementos existe una organización deductiva del conocimiento matemático. Esto es, con 23 definiciones, 5 nociones comunes, 5 postulados, ${ }^{23}$ más los diagramas, prueba Euclides en una secuencia deductiva las 48 proposiciones que componen este primer libro. ${ }^{24}$ Por otro lado, y principalmente en los Nueve capítulos, lo que se buscaba eran los principios guías (gang ji) de las matemáticas, esto es, una serie de procedimientos generales que permitieran resolver una variedad de problemas que pertenecieran a una misma categoría. Esto es, de los 246 problemas que se tratan en los Nueve capítulos, lo que el autor nos muestra es que cada uno de ellos es similar a una categoría de problemas, los cuales, se resuelven por uno o varios procedimientos generales - como el caso expuesto del capítulo 9, en el que se usa el procedimiento gou gu y lü para resolver los 24 problemas de una misma categoría- (Cullen 1996, p. 75; Chemla 2003; Lloyd 2009, p. 24). ${ }^{25}$

${ }^{22}$ Véase la nota 24.

${ }^{23}$ Esta presentación sigue la edición de Heiberg (1883-1885). Véase Heath 1956 (pp. 91-113) para una presentación general de las diferentes versiones y traducciones que se hicieron a lo largo de la historia.

${ }^{24}$ Las únicas excepciones a lo largo de los Elementos son las proposiciones en las que además de las definiciones, nociones comunes, postulados y diagramas se emplea también el método de la superposición, que consiste en el movimiento de una figura o línea sobre otra para establecer su coincidencia. Este método se usa solamente en tres pruebas, en I.4, I.8 y III.24. Una hipótesis de por qué esto ocurre es que Euclides evita recurrir a dicho método por su restricción de los movimientos permitidos al mínimo, asegurando así la fiabilidad de su práctica matemática (Heath 1956, p. 249; Mancosu 1996, pp. 28-33).

${ }^{25}$ Algunos autores han hablado, en este sentido, de la búsqueda de los procedimientos generales frente a la abstracción de los Elementos (Chemla 2003). Nosotros 
En relación con esta diferencia, es interesante ver el prefacio de Tseng - presentado y traducido por Swetz (1984) - a la edición revisada de los Elementos publicada en 1865 en China, donde dice,

de acuerdo con nuestras matemáticas tradicionales, cada sección deriva su nombre de una función (práctica específica). Los estudiantes siguen las reglas para resolver sus problemas. Usan las matemáticas durante toda su vida sabiendo solo cómo hacerlo y no por qué se hace. Por lo tanto, las consideran como una materia muy difícil porque están confundidos, sabiendo el método, pero no los principios [...] La geometría de Euclides (por otro lado) trata no con el método, sino con los principios. (pp. 171-173)

Por otro lado, observamos en el libro I de los Elementos que hay 48 diagramas, esto es, uno por cada proposición que se demuestra en este capítulo. Sin embargo, en el capítulo nueve de los Nueve capítulos hay 24 problemas, pero sólo cuatro diagramas fundamentales reducibles a dos. Este hecho está relacionado con la búsqueda de la generalización de los procedimientos en esta tradición, por lo que un mismo procedimiento podía servir para resolver una variedad de problemas y, por lo tanto, el mismo diagrama serviría para dilucidar por qué ese procedimiento es correcto para buscar la solución deseada. De esta manera, queda patente que las funciones de los diagramas son diferentes. En los Elementos los diagramas se utilizan para promover inferencias, mientras que los primeros comentaristas de la tradición china los usaron con una intención pedagógica, así como explicativa de los procedimientos involucrados en la resolución de los diferentes problemas.

Además, hemos podido ver que en la demostración de Euclides del teorema de Pitágoras es necesaria la posesión de algún conocimiento previo para llevarla a cabo, lo cual es característico de la estructura deductiva del conocimiento matemático de esta obra. Sin embargo, esto no ocurre en el caso del Zhou bi ni en los Nueve capítulos, como vimos en el caso de 9.1, donde se presentaba el procedimiento y diagrama sin hacer mención de ninguno de los procedimientos presentados en los capítulos anteriores. Este hecho está relacionado,

decimos deducción y no abstracción puesto que el hecho de que la abstracción se asocie a la geometría euclidiana no es algo aceptado unánimemente por los especialistas. Por ejemplo, Netz (1999) subraya la importancia de tomar en consideración la generalidad del uso de diagramas. Esto es, la posibilidad de poder volver a usar algo ya demostrado, como sería el caso de volver a usar lo demostrado en I.1 para otras líneas y segmentos (pp. 252-258). 
de nuevo, con la estrategia heurística de esta tradición, donde lo importante era precisamente mostrar cómo un conjunto de problemas similares, pertenecientes a una misma categoría, podían ser resueltos con un procedimiento determinado, acompañado por una serie de diagramas, y que no tenían por qué ser aplicados necesariamente a otros problemas de otras categorías.

En cuanto a la segunda categoría de diferencias, en la geometría euclidiana encontramos construcciones en las que el movimiento no es relevante. ${ }^{26}$ Sin embargo, en las obras de la matemática china aquí presentadas hemos podido observar que el movimiento es una parte esencial de la manipulación de dichos diagramas, donde los autores hablaban de cortar, pegar, colorear, etc. La diferencia se hace más patente si vemos, por ejemplo, el diagrama de la proposición I.2 (Figura 6) de los Elementos. Aquí, Euclides hace uso de un diagrama en el que se llevan a cabo diversas construcciones justificadas por diferentes postulados, nociones comunes, la proposición I.l probada anteriormente, etc., con el objetivo de demostrar que se puede construir una recta igual a una recta dada. Este tipo de problemas no tendría ni siquiera sentido en la práctica matemática china, puesto que como hemos visto, sería tan simple como mover una línea de un lado hacia otro, ya que se permitía cortar y pegar partes del diagrama.

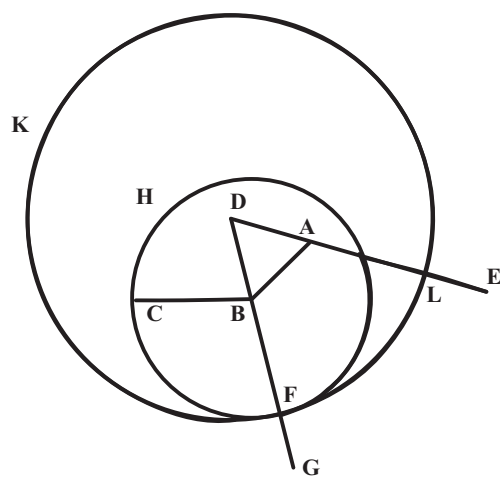

Figura 6. Diagrama usado en I.2. En esta prueba lo que se pide es que construyamos en un punto A - como extremo- una recta igual a una recta dada. En este caso, la recta $\mathrm{AL}$ se ha demostrado que es igual a la recta $\mathrm{BC}$.

${ }^{26}$ Véase la nota 24. 
Por otro lado, los diagramas euclidianos se pueden deformar hasta cierto punto, pues no es necesario que éstos exhiban los aspectos métricos de un modo preciso — justificados por la parte textual—, y la información co-exacta empleada en la prueba permanecería estable. Sin embargo, Zhao Shuang y Liu Hui han de realizar movimientos rígidos de partes del diagrama, manteniendo en todo momento la misma área o volumen para demostrar de manera fiable por qué determinado procedimiento es correcto. Por ejemplo, sería imposible mostrar por qué el procedimiento gou gu es correcto con el diagrama que estos dos comentaristas presentan (Figuras 4 y 5) si las áreas de las figuras involucradas en dicho diagrama se deformaran al ser movidas de un lugar a otro.

En cuanto a sus similitudes, encontramos, por un lado, que los diagramas fueron considerados como partes indispensables de ambas prácticas matemáticas, donde no se utilizaron como meras representaciones estáticas en las demostraciones matemáticas, sino que son utilizados como herramientas que han de ser manipuladas de acuerdo con las reglas y funciones que desempeñan dentro de su propia práctica matemática - con una función inferencial en Euclides, y explicativa de los algoritmos en las obras chinas-. En este sentido, en Euclides las letras se usan para conectar texto y diagrama, y en el caso de la matemática china tenemos un elemento análogo funcionalmente, que serían los colores. Por ejemplo, si vemos I.47 en Euclides (Figura 3) se afirma "dibujar AL paralelo a BD"; mientras que en el caso de los Nueve capítulos (Figura 5), se afirma "multiplicar la base por sí misma y obtenemos el cuadrado bermellón". En ambas presentaciones, estos elementos - letras y colores, respectivamente - ayudan a interpretar y manipular los diagramas de una manera adecuada. ${ }^{27}$

Con este análisis comparativo hemos querido mostrar cómo nuestro marco teórico puede ayudar a entender y analizar correctamente el desarrollo de diferentes prácticas matemáticas de la antigüedad. Por un lado, hemos propuesto una diferencia entre niveles de análisis, el cual, permite analizar correctamente las diferencias de las diversas prácticas matemáticas como un producto del desarrollo históricocultural de cada una. A pesar de las diferencias que hemos encontrado entre las obras presentadas, nuestro análisis contextual nos lleva a

${ }^{27}$ Respecto a este tema, Martzloff (1997, p. 22) señala el hecho de que cuando Matteo Ricci y Xu Guangqi tradujeron los seis primeros libros de los Elementos por primera vez - traducida como Yihe yuanben, esto es, "Elementos de geometría"-, tuvieron que introducir algunas innovaciones respecto a las figuras geométricas, pues éstas iban acompañadas de letras. Para ello, usaron las series denarias yi, bing, ding, etc., en lugar de colores, como habían hecho tradicionalmente. 
proponer la necesidad de revisar ciertas afirmaciones acerca de la poca importancia de las obras de la antigua civilización china frente a los Elementos - específicamente, aquellas concepciones que consideran a las primeras como meras colecciones de problemas concretos, en contraposición a la segunda, a la que se le reconoce como un cuerpo de conocimiento plenamente teórico- - Hemos comprobado en este trabajo que cada una de estas prácticas adoptaron estrategias diferentes, aunque tuvieron un objetivo común, el cual está relacionado con el establecimiento de resultados matemáticos lo suficientemente generales y precisos. De esta manera, podemos caracterizar a ambos cuerpos de conocimiento como plenamente matemáticos y situarlos a un nivel teórico de investigación matemática.

Para entender este punto, es necesario tener en cuenta los niveles de análisis que presentamos en la sección 2 de nuestro trabajo. Es decir, a un nivel de análisis macro podemos reconocer que estos dos desarrollos o prácticas matemáticas coinciden en ser consideradas como plenamente matemáticas, aunque siguieran estrategias o métodos de resolución de problemas diferentes. Lo habitual en la historia de las matemáticas, sin embargo, ha sido la consideración de que las matemáticas de la civilización china fueron inferiores a las de la civilización griega, precisamente por no seguir el camino deductivo característico de esta tradición. ${ }^{28}$ En este primer nivel, y siguiendo las definiciones tentativas de lo que sean las matemáticas que presentamos en la introducción, podríamos reconocer a ambas como matemáticas en un sentido pleno, ya que son prácticas dedicadas al estudio de números y/o configuraciones espaciales, usando para ello ciertos símbolos y/o herramientas, y que además estuvieron guiadas por los valores epistemológicos característicos de la investigación teórica en matemáticas, relacionados con la búsqueda de la generalidad y exactitud o precisión de los resultados alcanzados.

\section{Conclusiones}

En nuestro trabajo hemos asumido que, para analizar comparativamente desarrollos matemáticos de la antigüedad, es necesario hacerlo de acuerdo con algunas de las asunciones de la filosofía de las prácticas matemáticas. Además, hemos destacado la importancia de una

${ }^{28}$ Véase Cullen (2009, p. 592) para una crítica de este tipo de afirmaciones. Por otro lado, es interesante que cuando el matemático chino más prominente de los siglos XVII-XVIII leyó la traducción de los seis primeros libros de los Elementos afirmara que todos los métodos - excepto uno - de esta obra ya se encontraban en los Nueve capítulos (Xu 2005, p. 6). Por lo tanto, se podría afirmar que, obviando las diferencias en cómo estas matemáticas proceden, alcanzaron resultados similares. 
aproximación contextual, así como la división entre los niveles macro, meso y micro del análisis del conocimiento matemático.

De esta manera, hemos mostrado que los diagramas en los textos matemáticos analizados en nuestro trabajo pueden considerarse herramientas que han de ser manipuladas de acuerdo con una práctica compartida por los matemáticos que de ellas hacen uso. Estas herramientas son creadas con un propósito específico, permiten razonamientos diferentes — promoción de las inferencias en las matemáticas de Euclides, y con un papel pedagógico y explicativo en las matemáticas chinas_-, dado los problemas relevantes en la práctica en la cual los agentes se encuentran, sus objetivos, y las finalidades para las cuales tales herramientas fueron creadas y empleadas. Que su empleo fuera diferente está relacionado con las diferentes estrategias trazadas por cada una de estas prácticas en su búsqueda de resultados generales y exactos, ya sea estableciéndolos de manera deductiva, por un lado, o buscando procedimientos generales, por otro. Sin embargo, a pesar de estas diferencias, ambas prácticas estuvieron guiadas por metas y valores característicos de desarrollos plenamente teóricos en la elaboración del conocimiento geométrico.

Por otro lado, hemos argumentado que la práctica matemática en las obras chinas analizadas está guiada por una metodología heurística centrada en la búsqueda de la generalización de sus procedimientos. Este tipo de organización del conocimiento matemático sería distinto al que llevó a cabo Euclides, donde primaba la estructura deductiva del conocimiento. Sin embargo, hemos querido mostrar que estas diferencias no nos conducen a una minusvaloración de una de estas prácticas frente a la otra, sino que nos llevan precisamente a subrayar la importancia de entender y valorar en su propio contexto de desarrollo las diferentes prácticas matemáticas que surgieron en la antigüedad. Ambas son tradiciones matemáticas que, a pesar de sus diferencias en el uso de herramientas y establecimiento de los resultados, desarrollaron un conocimiento matemático valioso y relevante para sus propias civilizaciones, así como para los desarrollos matemáticos que se elaboraron posteriormente en cada una de estas tradiciones. ${ }^{29}$

${ }^{29}$ Queremos agradecer a José Ferreirós, Valeria Giardino, María de Paz y Abel Lassalle Casanave los valiosos consejos que nos dieron para elaborar y mejorar este trabajo. También queremos agradecer a los participantes del grupo de lectura de Filosofía de las Matemáticas de la Universidad de Sevilla, en el cual tuvimos ocasión de presentar y discutir nuestro trabajo. Por último, agradecer a Matheus Valente y Emiliano Boccardi por su lectura y comentarios. Este trabajo fue realizado con el apoyo financiero e institucional de la Fundação de Amparo à Pesquisa de São Paulo (FAPESP) a través del Proceso \#2016/20480-5. 


\section{BIBLIOGRAFÍA}

Artmann, B., 1999, Euclid -The Creation of Mathematics, SpringerVerlag, Nueva York.

Avigad, J., E. Dean, y J. Mumma, 2009, “A Formal System for Euclid's Elements", Review of Symbolic Logic, vol. 2, no. 4, pp. 700-768.

Bray, F., V. Dorofeeva-Lichtmann y G. Métailié (eds.), 2007, Graphics and Text in the Production of Technical Knowledge in China, Brill, Leiden, Boston.

Charette, F., 2012, "The Logical Greeks versus the Imaginative Oriental: on the Historiography of "Non-Western" Mathematics During the Period 1820-1920", en Chemla 2012, pp. 274-293.

Chemla, K. (ed.), 2012, The History of Mathematical Proofs in Ancient Traditions, Cambridge University Press, Nueva York.

, 2010, "Changes and Continuities in The Use of Diagrams $t u$ in Chinese Mathematical Writings (3rd century-14th century)", East Asian Science, Technology, and Society: An International Journal, vol. 4, no. 2, pp. 303-326.

, 2009, "On Mathematical Problems as Historically Determined Artifacts: Reflections Inspired by Sources from Ancient China", Historia Mathematica, vol. 36, no. 3, pp. 213-246.

— , 2003, "Generality above Abstraction: The General Expressed in Terms of the Paradigmatic in Mathematics in Ancient China", Science in Context, vol. 16, no. 3, pp. 413-458.

Chemla, K. y S. Guo, 2004, Les neuf chapitres: Le Classique mathématique de la Chine ancienne et ses commentaires, Dunod, París.

Cullen, C., 2009, "People And Numbers in Early Imperial China", en Robson and Stedall 2009, pp. 591-618.

—. 1996, Astronomy and Mathematics in Ancient China: The Zhou bi suan jing, Cambridge University Press, Cambridge.

Dauben, J.W., 2008, "Suan Shu Shu. A Book on Numbers And Computation", Archive for History of Exact Sciences, vol. 62, no. 2, pp. 91-178.

De Regt, H.W. y D. Dieks, 2005, "A Contextual Approach to Scientific Understanding", Synthese, vol. 144, no. 1, pp. 137-170.

Ferreirós, J., 2016, Mathematical Knowledge and the Interplay of Practices, Princeton University Press, Princeton.

Giardino, V., 2017, "Diagrammatic Reasoning in Mathematics", en Magnani y Bertolotti 2017, pp. 499-522.

— 2013, "A Practice-Based Approach to Diagrams", en Moktefi y Shin 2013, pp. 135-151.

Giardino, V. y R. Casati, 2013, "Public Representations and Indeterminacies of Perspectival Content", en Kondor 2013, pp. 111-126.

Giardino, V. y G. Greenberg, 2014, "Introduction: Varieties of Iconicity", Review of Philosophy and Psychology, vol. 6, no. 1, pp. 1-25. 
Hardy, G.H., 1940/1992, A Mathematician's Apology, Cambridge University Press, Cambridge.

Heath, T., 1956, The Thirteen Books of the Elements, Vol. I Books I and II, Cambridge University Press, Cambridge.

—_, 1921, A History of Greek Mathematics, Vol. 1: From Thales to Euclid, Dover.

Joseph, G.G., 2011, The Crest of The Peacock: Non-European Roots of Mathematics (3a. edición), Princeton University Press, Princeton, Oxford.

Kondor, Z. (ed.), 2013, Enacting Images - Representation Revisited, Herbert von Halem Verlag, Köln.

Lloyd, G.E.R., 2009, "What Was Mathematics in The Ancient World? Greek and Chinese Perspectives", en Robson y Stedall 2009, pp. 7-25.

Magnani, L. y T. Bertolotti (eds.), 2017, Springer Handbook of ModelBased Science, Springer Dordrecht, Heidelberg, Londres/Nueva York.

Mancosu, P., (ed.), 2008, The Philosophy of Mathematical Practice, Oxford University Press, Oxford, Nueva York.

- 1996, Philosophy of Mathematics and Mathematical Practice in the Seventeenth Century, Oxford University Press, Nueva York, Oxford.

Manders, K., 2008a, "Diagram-Based Geometric Practice", en Mancosu 2008 , pp. 65-79.

— 2008b, "The Euclidean Diagram”, en Mancosu 2008, pp. 80-133.

Martzloff, J.-C., 1997, A History of Chinese Mathematics, Springer, Heidelberg, Berlín.

Moktefi, A. y S.J. Shin (eds.), 2013, Visual Reasoning with Diagrams, Birkhäuser, Basel.

Netz, R., 1999, The Shaping of Deduction in Greek Mathematics: A Study of Cognitive History, Cambridge University Press, Cambridge.

Puertas Castaños, M.L. (trad.), 2000, Los Elementos de Euclides. Libros $I-I V$, Gredos, Madrid.

Robson, E. y J. Stedall (eds.), 2009, The Oxford Handbook of the History of Mathematics, Oxford University Press, Oxford, Nueva York.

Swetz, F., 1984, "The Introduction of Mathematics in Higher Education in China, 1865-1887", Historia Mathematica, vol. 1, no. 2, pp. 167-179.

Volkov, A., 2007, "Geometrical Diagrams in Traditional Chinese Mathematics", en F. Bray, V. Dorofeeva-Lichtmann y G. Métailié, pp. 425-459.

Wagner, R., 2017, Making and Breaking Mathematical Sense, Princeton University Press, Princeton, Oxford.

Xu, Y., 2005, "The First Chinese Translation of The Last Nine Books of Euclid's Elements and Its Source", Historia Mathematica, vol. 32, no. 1, pp. 4-32.

Recibido el 1 de marzo de 2018; revisado el 27 de junio de 2019; aceptado el 28 de junio de 2019. 